\title{
BMJ Open Oral cannabinoid-rich THC/CBD cannabis extract for secondary prevention of chemotherapy-induced nausea and vomiting: a study protocol for a pilot and definitive randomised double-blind placebo-controlled trial (CannabisCINV)
}

Antony J Mersiades, ${ }^{1}$ Annette Tognela, ${ }^{2}$ Paul S Haber, ${ }^{3,4}$ Martin Stockler, ${ }^{1,5}$ Nicholas Lintzeris, ${ }^{3,6}$ John Simes, ${ }^{1,7}$ Iain McGregor, ${ }^{8}$ Ian Olver, ${ }^{9}$ David J Allsop, ${ }^{8}$ Craig Gedye, ${ }^{10}$ Adrienne C Kirby, ${ }^{1}$ Rachael L Morton, ${ }^{1}$ Peter Fox, ${ }^{11}$ Stephen Clarke, ${ }^{12,13}$ Karen Briscoe, ${ }^{14}$ Morteza Aghmesheh, ${ }^{15}$ Nicole Wong, ${ }^{1}$ Anna Walsh, ${ }^{1}$ Carmel Hahn, ${ }^{7}$ Peter Grimison ${ }^{1,7}$

To cite: Mersiades AJ, Tognela A, Haber PS, et al. Oral cannabinoid-rich THC/CBD cannabis extract for secondary prevention of chemotherapyinduced nausea and vomiting: a study protocol for a pilot and definitive randomised doubleblind placebo-controlled trial (CannabisCINV). BMJ Open 2018;8:e020745. doi:10.1136/ bmjopen-2017-020745

- Prepublication history for this paper is available online. To view these files, please visit the journal online (http://dx.doi. org/10.1136/bmjopen-2017020745).

Received 31 December 2017 Revised 7 June 2018 Accepted 21 June 2018

Check for updates

(C) Author(s) (or their employer(s)) 2018. Re-use permitted under CC BY-NC. No commercial re-use. See rights and permissions. Published by BMJ.

For numbered affiliations see end of article.

Correspondence to Dr Antony J Mersiades; antony.mersiades@gmail.com

\section{ABSTRACT}

Introduction Chemotherapy-induced nausea and vomiting (CINV) remains an important issue for patients receiving chemotherapy despite guidelineconsistent antiemetic therapy. Trials using delta-9tetrahydrocannabinol-rich (THC) products demonstrate limited antiemetic effect, significant adverse events and flawed study design. Trials using cannabidiol-rich (CBD) products demonstrate improved efficacy and psychological adverse event profile. No definitive trials have been conducted to support the use of cannabinoids for this indication, nor has the potential economic impact of incorporating such regimens into the Australian healthcare system been established. CannabisCINV aims to assess the efficacy, safety and cost-effectiveness of adding TNTC11M, an oral THC/CBD extract to guideline-consistent antiemetics in the secondary prevention of CINV.

Methods and analysis The current multicentre, 1:1 randomised cross-over, placebo-controlled pilot study will recruit 80 adult patients with any malignancy, experiencing CINV during moderate to highly emetogenic chemotherapy despite guideline-consistent antiemetics. Patients receive oral TN-TC11M (THC 2.5mg/CBD $2.5 \mathrm{mg}$ ) capsules or placebo capsules three times a day on day -1 to day 5 of cycle $A$ of chemotherapy, followed by the alternative drug regimen during cycle $B$ of chemotherapy and the preferred drug regimen during cycle $\mathrm{C}$. The primary endpoint is the proportion of subjects attaining a complete response to CINV. Secondary and tertiary endpoints include regimen tolerability, impact on quality of life and health system resource use. The primary assessment tool is patient diaries, which are filled from day -1 to day 5 . A subsequent randomised placebo-controlled parallel phase III trial will recruit a further 250 patients.

\section{Strengths and limitations of this study}

- Largest and most definitive randomised double-blind placebo-controlled trial of cannabis for secondary prevention of chemotherapy-induced nausea and vomiting (CINV).

- First study to assess the impact on heath resource use and cost-effectiveness of cannabis within the Australian health system.

- Utility of patient-reported outcomes.

- Self-titrating dose of tetrahydrocannabinol/ cannabidiol.

- Primary outcome measure (complete response) does not include nausea assessment, to ensure comparability with other CINV trials.

Ethics and dissemination The protocol was approved by ethics review committees for all participating sites. Results will be disseminated in peer-reviewed journals and at scientific conferences.

Drug supply Tilray.

Protocol version 2.0, 9 June 2017.

Trial registration number ANZCTR12616001036404; Pre-results.

\section{INTRODUCTION}

Chemotherapy-induced nausea and vomiting (CINV) remains a significant cause of morbidity in oncology patients despite the best current antiemetic prophylaxis. ${ }^{1}$

The Multinational Association of Supportive Care in Cancer (MASCG) has 
published guidelines which recommend a standardised regimen of a 5- $\mathrm{HT}_{3}$ receptor antagonist, with dexamethasone, and for most regimens an NK-1 antagonist for optimal antiemetic prophylaxis of moderate or high emetic risk. ${ }^{2}$ Even among patients receiving guideline-consistent antiemetic prophylaxis, contemporary observational studies of moderate or highly emetogenic chemotherapy found that $46 \%-57 \%$ experienced significant nausea and 9\%-37\% experienced vomiting. ${ }^{3-6}$

\section{Cannabinoids for CINV}

The mechanism of delayed nausea and vomiting is incompletely understood, but may involve non-serotonergic receptors including the cannabinoid $\mathrm{CB}_{1}$ receptor, with a potential role for cannabis products in its amelioration. ${ }^{7}$ There is limited evidence of the efficacy of cannabis products for the prevention and treatment of CINV. ${ }^{8}$ While several studies have been carried out using smoked marijuana or synthetic oral tetrahydrocannabinol medicines (THC: dronabinol, nabilone) for treating CINV, most showed limited efficacy, were inadequately powered and used outdated control antiemetic arms. ${ }^{9}$

The major limitations of current oral THC-rich cannabinoids for this indication are unpredictable gastrointestinal absorption, poor bioavailability, delayed onset of action and inability to rapidly self-titrate the dose for nausea control depending on tolerance. ${ }^{9}$ Furthermore, THC-only medications can be limited in their therapeutic potential by their intoxicating and disorientating central nervous system effects at the higher doses often needed to control nausea and vomiting. ${ }^{9}$ Cannabidiol (CBD) is known to counteract the psychiatric adverse effects of THC, and has inherent anxiolytic properties. ${ }^{10}$ The addition of CBD to THC should ameliorate the intoxicating effects of THC, and allow delivery of higher therapeutic doses, without increasing the overall intoxication, paranoia and euphoria associated with THC, with diminished potential for abuse. ${ }^{11}$

Nabiximols (Sativex; GW Pharmaceuticals, UK) is a THC/CBD cannabis extract derived from the Cannabis sativa plant containing THC and CBD in defined and near-equal amounts, and presented as a buccal spray. ${ }^{11}$ A published small pilot double-blind randomised trial of nabiximols for secondary prevention of CINV found substantial efficacy, high patient acceptability and manageable side effects. ${ }^{12}$ In this study, 16 patients who experienced CINV after moderately emetogenic chemotherapy, despite prophylaxis with a guideline-consistent antiemetic regimen, ${ }^{12}$ were randomised to nabiximols or placebo. Participants received up to $8.1 \mathrm{mg}$ THC/7.5 mg CBD over 2 hours, then up to $21.6 \mathrm{mg}$ THC/ $20 \mathrm{mg}$ CBD every 24 hours for $5 \times 24$-hour periods. In the nabiximols group, the mean dose per 24 hours was $13 \mathrm{mg}$ THC (range 5.4-13.5)/12 mg CBD (range 5-12.5) and the median duration was 3 days (range $1-5)$. The short dose titration was tolerated in $86 \%$ receiving nabiximols, with one patient discontinuing because of transient psychiatric effects. Adverse effects were experienced in $86 \%$ receiving nabiximols and $67 \%$ receiving placebo. Substantial efficacy was observed, with complete response in $71 \%$ vs $22 \%$, (difference $49 \%, 95 \%$ CI $1 \%$ to $75 \%$ ), absence of delayed emesis in $71 \%$ vs $22 \%$ and nausea in $57 \%$ vs $22 \%$, however no difference in acute nausea nor vomiting. ${ }^{12}$ Nabiximols is licensed for the indication of neurospasticity in multiple sclerosis, but is not commercially available in Australia, and is not available for use in our proposed trial. There is a paucity of data on the cost-effectiveness of nabiximols outside of multiple sclerosis. ${ }^{13}$

This study will use a novel oral THC/CBD capsule formulation ('TN-TC11M', Tilray). The product is intended to provide equivalent systemic exposures to THC and CBD as nabiximols and was formulated for a consistent and reproducible pharmacokinetic profile, allowing patients to self-titrate as needed without a concern for a delayed onset. The product will be dosed similarly to the Duran study, commencing 24 hours prior to chemotherapy to confirm tolerability.

\section{HYPOTHESIS}

\section{Primary hypothesis}

We hypothesise that the addition of TN-TC11M to standard guideline-based antiemetics will improve the control of CINV, leading to improved quality of life. The potential resource implications are of higher CINV regimen drug costs, but reduced costs of the management of refractory CINV through the reduced need for rescue medications and reduced hospitalisation.

\section{METHODS AND ANALYSIS}

The cannabis for CINV trial is an Australian-based, double-blind, placebo-controlled, two-stage randomised controlled trial. The primary aim is to determine the efficacy of adding an oral CBD-rich THC extract (TN-TC11M) for secondary prevention of CINV after failure of a guideline-consistent prophylactic antiemetic regimen.

\section{Study objectives}

The primary objective of this study is to compare, among patients randomised to TN-TC11M or placebo, the ability to control emesis and nausea, as determined by:

- The proportion of patients achieving a 'complete response' during the overall phase of treatment (0-120 hours), defined as no emesis and no use of rescue medications.

The pilot study (crossover) and definitive trial (parallel) will use different methods of analysis as outlined in the statistical analysis section.

The secondary objectives are to compare, among patients randomised to TN-TC11M or placebo:

\section{- Efficacy}

- Proportions of patients during acute (0-24hours), delayed (24-120 hours) and overall (0-120 hours) 
phases of cycles A, B and C with (1) complete response, (2) no emesis (vomiting or dry retching), (3) no significant nausea, defined as degree of nausea $<2$ out of 10 using an 11-point rating scale and (4) no use of rescue medications.

- Number of emetic episodes during 0-120 hours of cycles A, B and C.

- Cannabinoid-related and other adverse events

- Endpoint: Proportions with specified cannabinoid-related and other adverse events (structured checklist, Common Terminology Criteria for Adverse Events (CTCAE).

- Health-related quality of life

- Endpoint: Summary scales (nausea, vomiting) of Functional Living Index-Emesis (FLIE) and all items of Assessment of Quality of Life (AQOL-8D).

- Regimen's acceptability

- Endpoint: Adherence (patient diaries, pill counts), preference.

The tertiary and correlative objectives are:

- To compare health system resource use and costs between randomised groups

- Endpoint: Health system resource use and costs.

- To model the cost-effectiveness of cannabinoid therapy compared with placebo or other antiemesis alternatives, for prevention of CINV, from the perspective of the healthcare system

- Endpoint: Health outcomes relative to costs.

\section{Trial oversight and monitoring}

The CannabisCINV trial is a collaboration between the Chris O'Brien Lifehouse; the National Health and Medical Research Council (NHMRC) Clinical Trials Centre, University of Sydney; and the Lambert Initiative for Cannabinoid Therapeutics, University of Sydney.

The University of Sydney is the study sponsor. The NHMRC Clinical Trials Centre will be responsible for trial coordination, monitoring, site audits, management, data acquisition and statistical analysis.

Safety and feasibility endpoints will be reviewed at 3 monthly intervals by the independent data and safety monitoring committee (IDSMC) during both the pilot and phase III trial. The IDSMC will assess the rate of serious adverse events (SAEs) (graded $\geq 3$ by CTCAE criteria) and accrual, with no pause in recruitment planned. Consideration will be given to altering aspects of the study if:

- The accrual rate is insufficient to complete the study in a reasonable time frame.

- The rate of SAEs in the oral TN-TC11M arm is unacceptably high compared with the placebo arm.

- Medical or ethical reasons emerge affecting continued performance of the study.

Protocol amendments can only be made by the trial management committee and must be approved by the institutional Human Research Ethics Committee (HREC) prior to implementation.

\section{Patient and public involvement}

Consumer representatives were involved in the development of this trial. The primary and secondary outcomes of this study, including adverse events, are patient-reported outcomes, emphasising the focus of this study on patient priorities, experience and preferences. Results of this study will be disseminated to study participants through peer-reviewed journals and at scientific conferences.

\section{Trial design}

The protocol consists of a pilot phase II, double-blinded, randomised, placebo-controlled cross-over study followed by a planned definitive phase III, blinded, randomised, parallel, placebo-controlled trial (see figure 1). The pilot trial will be conducted at 10 sites across the state of New South Wales (NSW), Australia, in both tertiary referral centres and regional centres in outpatient comprehensive cancer care units. A list of the study sites can be accessed from the Australian New Zealand Clinical Trials Registry.

\section{Inclusion criteria}

Patients who fulfil all of the following characteristics will be considered eligible for enrolment:

- Adult patients with any malignancy.

- Receiving moderate to highly emetogenic intravenous chemotherapy (day 1) in a 14-day or 21-day cycle.

- Experienced significant CINV, defined as requiring $\geq 1$ dose of rescue medication for vomiting or distress by nausea, and/or $\geq$ moderate nausea on a five-point rating scale $(0=$ nil, $1=$ minimal, $2=$ moderate, $3=$ severe, $4=$ very severe), at any time during the current chemotherapy regimen despite guideline-consistent antiemetics.

- Planned for at least two further cycles of the same chemotherapy regimen.

- Eastern Cooperative Oncology Group (ECOG) performance status of 2 or less.

\section{Exclusion criteria}

Patients with the following characteristics will be excluded from study enrolment:

- Symptomatic central nervous system/leptomeningeal disease, gastrointestinal tract obstruction or disease-related nausea or vomiting requiring daily antiemetic therapy.

- Oral chemotherapy during study treatment (eg, oral capecitabine).

- Radiotherapy to the brain, abdomen or pelvis within the week prior to commencing study treatment, or planned during study treatment.

- Contraindication to cannabinoid treatment:

- Unstable cardiovascular disease (uncontrolled hypertension, unstable ischaemic heart disease, unstable congestive cardiac failure).

- History of epilepsy or recurrent seizures.

- History of schizophrenia, other psychotic illness, severe personality disorder, suicidal ideation or other 


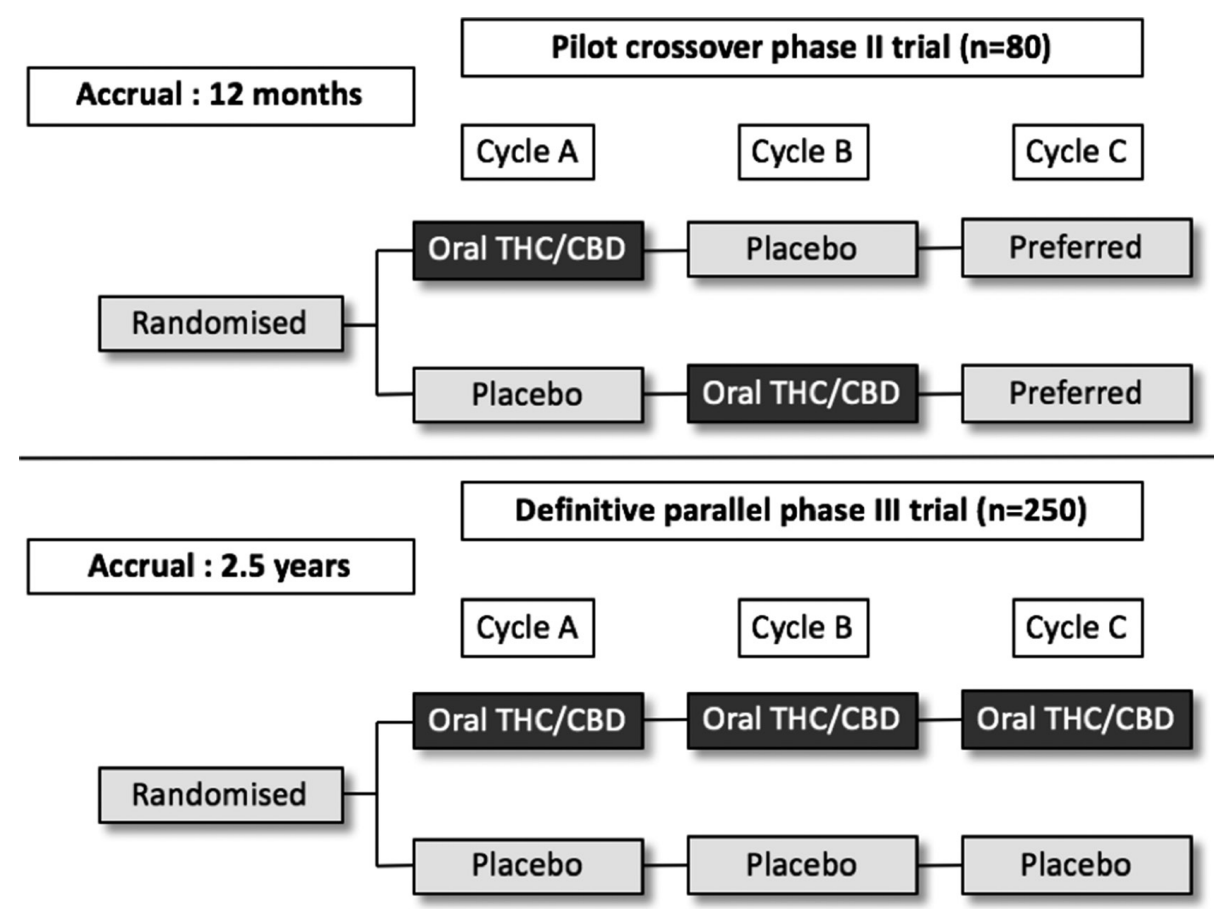

Figure 1 Study schema for pilot phase II, double-blinded, randomised, placebo-controlled cross-over study and planned definitive phase III, blinded, randomised, parallel, placebo-controlled trial. CBD, cannabidiol; THC, tetrahydrocannabinol.

significant psychiatric disorder, other than depression associated with the underlying condition.

- Substance use disorder (International Classification of Diseases (ICD-10) criteria of abuse or dependence) to alcohol, opioids, benzodiazepines, cannabis or illicit stimulants.

- Unwilling/unable to avoid driving or operating heavy machinery during and for up to 72 hours after taking study medication.

- Concerns regarding safe storage of medication.

- Cannabis or cannabinoid-based medications within 30 days of study or unwilling to abstain for the duration of the study.

- Prior hypersensitivity or intolerable adverse reaction to cannabis or cannabinoid-based medications, 5HT3 antagonist, dexamethasone or NK1 antagonist.

- Pregnancy, lactation or inadequate contraception. Women must be postmenopausal, infertile or use a reliable means of contraception. Women of childbearing potential must have a negative pregnancy test done within 7 days prior to registration. Men must have been surgically sterilised or use a (double if required) barrier method of contraception.

\section{Investigational medical product}

TN-TC11M

This study will use a novel oral THC/CBD capsule formulation ('TN-TC11M', Tilray) derived from Cannabis sativa $L$. extract. It contains delta-9-THC and CBD in a 1:1 ratio, with each oral capsule containing $2.5 \mathrm{mg}$ THC and $2.5 \mathrm{mg}$ CBD. This is intended to provide equivalent systemic exposures to THC and CBD as those obtained from nabiximols (a single dose of nabiximols which comprises $2.7 \mathrm{mg}$ THC and $2.5 \mathrm{mg} \mathrm{CBD}$ ). In contrast to nabiximols, TN-CT11M was formulated for a consistent and reproducible pharmacokinetic profile, allowing patients to self-titrate as needed without a concern for a delayed onset. Investigational product will be dosed similarly to the Duran study, ${ }^{12}$ and administration will commence 24 hours prior to chemotherapy to confirm tolerability.

\section{Placebo}

The placebo will be in white capsules, identical in appearance to that of the active treatment.

\section{Randomisation, allocation concealment and double-blind} conditions

Randomisation will be performed centrally using minimisation, with stratification by chemotherapy emetogenic risk (moderate vs high by MASCC criteria) and by site.

Pharmaceutical Packaging Professionals will store and distribute the drug on behalf of the study sponsor to all participating sites on receipt of all drug order forms.

\section{Background treatment}

All patients will receive antiemetics including 5HT3 antagonist, corticosteroid and (where indicated) NK1 antagonist; according to a prespecified choice of regimen consistent with guidelines from eviQ Cancer Treatments Online $^{14}$ and/or MASCC guidelines for optimal antiemetic prophylaxis of chemotherapy. ${ }^{2}$

The following rescue therapies are permitted:

- Lorazepam, for example, $1 \mathrm{mg}$ per oral (PO) two times a day when required (prn). 
- Metoclopramide, for example, $10 \mathrm{mg}$ PO three times a day prn.

- Haloperidol, for example, 0.5-1 mg PO three times a day prn.

- Prochlorperazine, for example, 5-10 mg PO three times a day prn, $25 \mathrm{mg}$ sup per rectum every 8 hours prn.

- Olanzapine, for example, $5 \mathrm{mg}$ PO two times a day or $10 \mathrm{mg}$ PO mane for 3 days.

Note:

- 5HT3 antagonists should only be used when other rescue therapies have failed.

\section{Recruitment and consent}

Patient screening and enrolment undertaken at participating sites will be overseen by the site principal investigator and performed by trained study personnel. A screening log will document all eligible patients screened and approached along with reasons for any exclusions. Patients will provide written informed consent prior to study enrolment.

\section{Pilot study}

The phase II pilot trial $(\mathrm{n}=80)$ will be a multisite, 1:1 randomised, double-blind, placebo-controlled trial (figure 1). The dosing regimen will be similar to that used in the nabiximols study by Duran et al described above, which was shown to be well tolerated and effective, however, patients will also receive study drug for 24 hours prior to chemotherapy to confirm tolerability. It will use a cross-over design and a significance level of $10 \%$ to increase efficiency, and allow patients to nominate a preferred drug in the final cycle.

In the pilot study, during chemotherapy cycle A, subjects will be randomised to receive oral TN-TC11M capsules $(2.5 / 2.5 \mathrm{mg})$ three times a day from day -1 to 5 or identical placebo capsules, in addition to guideline-consistent antiemetics. For cycle B, subjects will crossover to receive the alternative formulation in the same schedule.

On treatment, patients will be able to self-titrate their TN-TC11M/placebo exposure based on tolerance, with a maximum does of $30 \mathrm{mg} / 30 \mathrm{mg}$ per day (12 tablets) (table 1). Following cycle $\mathrm{B}$, subjects are asked to nominate their preferred regimen after cycle $\mathrm{B}$, and where relevant will receive that agent with cycle $\mathrm{C}$.

\section{Definitive study}

The definitive randomised phase III study $(\mathrm{n}=250)$ will assess the efficacy of the addition of TN-TC11M to guideline consistent antiemetics as secondary prevention of CINV. It will have a parallel group design, to reduce bias given the possibility of carry-over effect from cross-over in subsequent cycles, and to investigate longer-term efficacy over multiple chemotherapy cycles.

In the definitive study, during cycle A, subjects will receive oral TN-TC11M capsules $(2.5 / 2.5 \mathrm{mg})$ three times a day from day -1 to 5 or identical placebo capsules, in the same treatment schedule (see table 1). For cycle B (and C if relevant), subjects will recommence treatment with the investigational product on their maximum tolerated dose from the previous cycle, with further scope to self-titrate according to symptoms.

\section{Data acquisition}

The participant will be instructed on the use of the patient diary, which has been designed for this study, to record:

- Nausea (past 24-hour period), recorded using an 11-point rating scale.

- Date, time and type of rescue medication used.

- Vomiting and dry retching episodes.

The patient diary will be completed by the patient. There will be daily assessment of patients on days 1-6 of each cycle to ensure:

- Study treatments are taken appropriately.

- Accurate records in the patient diary.

- Completion of structured checklist of cannabinoid-specific adverse events.

- Advice is provided regarding management of any adverse events.

Trial data will be recorded on the electronic Case Report Forms (eCRFs) provided and will monitored by clinical trials staff from the NHMRC Clinical Trials Centre.

\section{Unblinding and poststudy care}

Following completion of trial treatment, patients who have experienced benefit and require ongoing treatment with the same chemotherapy regimen may be eligible for ongoing access to TN-TC11M, free of charge, contingent on unblinding performed by an unblinded statistician.

\section{STATISTICAL CONSIDERATIONS \\ Sample size estimation}

The estimated sample size for the pilot trial is 80 patients (40 per arm), using a primary endpoint of complete response to the study drug and placebo during cycle A and $\mathrm{B}$ of treatment. Using a cross-over design, randomising patients to either study drug followed by placebo or placebo followed by study drug, will have $80 \%$ power at a two-sided significance level of $10 \%$ to detect a $20 \%$ difference in discordant responses (response on one intervention, but not the other). Accrual is expected to take 12 months. The $20 \%$ difference is based on the assumption that $42 \%$ of patients on the study drug will respond compared with $22 \%$ on placebo, and that the responders in the placebo group will respond/not respond equally on the study drug ( $11 \%$ respond on each).

The estimated sample size for the definitive trial is 250 patients (125 per arm), using a primary endpoint of complete response during cycle A of study treatment. A sample size of 250 patients provides $80 \%$ power at two-sided $5 \%$ level of significance to detect improvement in complete response from $22 \%$ to $42.5 \%$. Accrual is expected to take 2.5 years.

The sample size for each trial will allow for a drop-out/ ineligibility rate of $20 \%$. 
Table 1 On treatment schedule with dose modifications

\begin{tabular}{|c|c|c|c|c|}
\hline Day & Time & Dose & Dose titration & $\begin{array}{l}\text { Maximum dose per } \\
\text { 24-hour period }\end{array}$ \\
\hline \multirow{3}{*}{-1} & Mane & One capsule & NA & \multirow{3}{*}{$\begin{array}{l}\text { Five capsules } \\
12.5 \mathrm{mg} \mathrm{THC} / \mathrm{CBD} \\
\text { or placebo }\end{array}$} \\
\hline & $\begin{array}{l}\text { Midi } \\
\text { (at least } 4 \text { hours } \\
\text { after previous dose) }\end{array}$ & Two capsules & Miss dose if intoxicated & \\
\hline & $\begin{array}{l}\text { Nocte } \\
\text { (at least } 4 \text { hours after } \\
\text { previous dose) }\end{array}$ & $\begin{array}{l}\text { One to two } \\
\text { capsules }\end{array}$ & $\begin{array}{l}\text { Miss dose if intoxicated } \\
\text { Reduce dose by one capsule if previous dose } \\
\text { not tolerated } \\
\text { Maitain dose if previous dose tolerated }\end{array}$ & \\
\hline \multirow[b]{2}{*}{1} & $\begin{array}{l}60 \text { min prior to } \\
\text { chemotherapy }\end{array}$ & $\begin{array}{l}\text { One to two } \\
\text { capsules }\end{array}$ & \multirow{2}{*}{$\begin{array}{l}\text { Miss dose if intoxicated } \\
\text { Reduce dose by one capsule if previous dose } \\
\text { not tolerated } \\
\text { Miss dose if previous dose tolerated } \\
\text { Increase dose by one capsule if previous dose } \\
\text { tolerated, but inadequate nausea control }\end{array}$} & \multirow{2}{*}{$\begin{array}{l}\text { Eight capsules } \\
20 \mathrm{mg} \text { THC/CBD } \\
\text { or placebo }\end{array}$} \\
\hline & $\begin{array}{l}\text { Immediately after } \\
\text { completion of day } 1 \\
\text { chemotherapy infusion }\end{array}$ & $\begin{array}{l}\text { One to three } \\
\text { capsules }\end{array}$ & & \\
\hline \multirow{3}{*}{$\begin{array}{l}2 \\
3 \\
4\end{array}$} & $\begin{array}{l}\text { Continue three times a day } \\
\text { Mane }\end{array}$ & $\begin{array}{l}\text { One to four } \\
\text { capsules }\end{array}$ & \multirow{3}{*}{$\begin{array}{l}\text { Miss dose if intoxicated } \\
\text { Reduce dose by one capsule if previous dose } \\
\text { not tolerated } \\
\text { Maintain dose if previous dose tolerated } \\
\text { Increase dose by one capsule if previous dose } \\
\text { tolerated, but inadequate nausea control }\end{array}$} & \multirow{3}{*}{$\begin{array}{l}\text { Twelve capsules } \\
30 \mathrm{mg} \text { THC/CBD } \\
\text { or placebo }\end{array}$} \\
\hline & Midi & & & \\
\hline & Nocte & & & \\
\hline 5 & $\begin{array}{l}\text { Mane } \\
\text { Midi (final dose) }\end{array}$ & $\begin{array}{l}\text { One to four } \\
\text { capsules }\end{array}$ & $\begin{array}{l}\text { Miss dose if intoxicated } \\
\text { Reduce dose by one capsule if previous dose } \\
\text { not tolerated } \\
\text { Maintain dose if previous dose tolerated } \\
\text { Increase dose by one capsule if previous dose } \\
\text { tolerated, but inadequate nausea control }\end{array}$ & $\begin{array}{l}\text { Eight capsules } \\
20 \mathrm{mg} \text { THC/CBD } \\
\text { or placebo }\end{array}$ \\
\hline
\end{tabular}

Table 1 shows the starting dose will be one capsule PO on the morning of day -1 , with the next scheduled dose of two capsules (if tolerated, at midday), followed by two capsules that evening (if tolerated). On day 1 , subjects receive one to two capsules $60 \mathrm{~min}$ prior to chemotherapy infusion, followed by one to three capsules immediately after completion of day 1 infusional chemotherapy, then one to three capsules 4 hours after completion of day 1 infusional chemotherapy. On days $2-5$, subjects take one to four tablets three times a day, with instruction to dose titrate according to intoxication, tolerance of prior dose and nausea control. The final dose is at midday on day 5 , with no further treatment until day -1 of the next chemotherapy cycle.

$\mathrm{CBD}$, cannabidiol; NA, not applicable; $\mathrm{TCH}$, tetrahydrocannabinol.

The current evidence suggests that this level of improvement is both worthwhile and feasible.

\section{Statistical analysis}

Pilot study

Analyses will be conducted using intention-to-treat (ITT) principles. The primary analyses will include all patients who were randomised. Missing values will not be imputed. The results will be examined for differential effect in the two periods and if necessary, the data from cycle A will be analysed separately.

The primary outcome is complete response. The primary analysis will be a comparison of the proportion of patients with a complete response between the two treatments over cycle A and cycle B, using McNemar's test. In the event that there is differential drop-out between period or a period by treatment interaction, the results from the first period will be analysed using a $\chi^{2}$ test. Analyses to assess period by treatment interaction will use generalised estimating equations (GEEs) to account for the correlation within a patient. Secondary analyses to adjust for any baseline variables will also use GEEs. All tests will use a two-sided significance level of $10 \%$.

\section{Definitive study}

The primary analyses will include all randomised patients using ITT principles, and compare the proportion of patients with complete response in the two treatment arms during the overall phase (0-120 hours) of cycle A, using a $\chi^{2}$ test. Secondary analyses adjusting for baseline variables will use GEEs. Binary secondary outcomes will be analysed as for the primary outcome. Count data will be analysed using a generalised linear 
model with a Poisson distribution. Cannabinoid-related adverse events and measures of adherence will be analysed with a $\chi^{2}$ test or two-sample t-test, or just listed depending on numbers. All tests will use a two-sided significance level of $5 \%$.

\section{Health economic analysis}

A within-trial and modelled economic evaluation will be undertaken to determine the incremental cost-effectiveness of oral cannabinoid therapy compared with placebo and other antiemetic therapies, from a health system perspective.

For the within-trial analysis, resource use will be identified and measured from trial case report forms (for hospitalisations), and through linkage to Medicare claims data for outpatient visits (Medicare Benefits Schedule) and prescribed medicines (Pharmaceutical Benefits Scheme). Australian unit costs will be applied to the resource usage data (eg, Australian Refined Diagnostic Related Groups and Medicare scheduled fees using the most recent reference year). For the modelled evaluation, resource use will be taken from the trial data for cannabinoid and placebo therapies, and supplemented with published estimates of resource use and costs for alternative 'usual care' antiemetic therapies.

The definitive study will employ a health economic analysis which will use the proportion of patients with 'complete response' (ie, participants with no emesis and no use of rescue medications, consistent with the primary endpoint); quality of life as measured by the FLIE with 5-day recall and AQOL-8D instruments, ${ }^{15}$ and quality-adjusted survival. Quality-adjusted survival time will be used to quantify the incremental effectiveness of cannabinoid treatment. Quality-adjusted survival will be calculated by applying utility weights for quality of life derived from the AQOL-8D utility instrument to survival data using established methods. In addition, we will conduct a sensitivity analysis to determine the incremental costs to achieve an outcome of no significant nausea, no emesis and no use of rescue medications.

Two cost-effectiveness outcomes will be reported: (1) the incremental cost per additional complete responder (trial primary endpoint) at 30 days after last dose of study drug and (2) the incremental cost per quality-adjusted life year (QALY) gained at 12 months, using extrapolated data. These will be expressed as incremental cost-effectiveness ratios and plotted on a cost-effectiveness plane. Bootstrapping will be used to estimate a distribution around costs and health outcomes, and to calculate CIs around incremental cost-effectiveness ratios. ${ }^{16} \mathrm{~A}$ cost-effectiveness acceptability curve will be plotted, providing information about the probability that the intervention is cost-effective given the Australian government's willingness to pay for a QALY gained. ${ }^{17}$ One-way, two-way and probabilistic sensitivity analyses will be undertaken for relevant variables, including cost of the study drug.

\section{ETHICS AND DISSEMINATION}

In Australia, the study will be conducted according to the Note for Guidance on Good Clinical Practice (CPMP/ ICH/135/95) annotated with Therapeutic Goods Administration Drug Safety and Evaluation Branch comments (July 2000) and in compliance with applicable laws and regulations. The study will be performed in accordance with the NHMRC Statement on Ethical Conduct in Research Involving Humans 2007-updated May 2015, the NHMRC Australian Code for the Responsible Conduct of Research 2007 and the principles laid down by the World Medical Assembly in the Declaration of Helsinki 2008.

To this end, no patient will be recruited to the study until all the necessary approvals have been obtained and the patient has provided written informed consent. Further, the investigator shall comply with the protocol, except when a protocol deviation is required to eliminate immediate hazard to a participant. In this circumstance the NHMRC CTC, principal investigator and HREC must be advised immediately.

\section{TRIAL STATUS}

Patient enrolment for the pilot study commenced in November 2016 at the Chris O'Brien Lifehouse in NSW, Australia, with the 10th NSW site opened in June 2017. To date, 49 patients have been enrolled, with anticipated pilot study enrolment completion by the third quarter 2018.

\section{Author affiliations}

${ }^{1}$ NHMRC Clinical Trials Centre, University of Sydney, Sydney, New South Wales, Australia

${ }^{2}$ Macarthur Cancer Therapy Centre, Campbelltown Hospital, Sydney, New South Wales, Australia

${ }^{3}$ Discipline of Addiction Medicine, University of Sydney, Sydney, New South Wales, Australia

${ }^{4}$ Drug Health Services, Sydney Local Health District, Sydney, New South Wales, Australia

${ }^{5}$ Department of Medical Oncology, Concord Cancer Care Centre, Concord Hospital, Sydney, New South Wales, Australia

${ }^{6}$ Drug and Alcohol Services, South East Sydney Local Health District, Sydney, New South Wales, Australia

${ }^{7}$ Department of Medical Oncology, Chris O'Brien Lifehouse, Sydney, New South Wales, Australia

${ }^{8}$ Lambert Initiative for Cannabinoid Therapeutics, University of Sydney, Sydney, New South Wales, Australia

${ }^{9}$ University of South Australia Cancer Research Institute, University of South Australia, Adelaide, South Australia, Australia

${ }^{10}$ Department of Medical Oncology, Calvary Mater Newcastle, Newcastle, New South Wales, Australia

${ }^{11}$ Central West Cancer Care Centre, Orange Health Service, Orange, New South Wales, Australia

${ }^{12}$ Department of Medical Oncology, Royal North Shore Hospital, Sydney, New South Wales, Australia

${ }^{13}$ Bill Walsh Cancer Research Laboratory, Kolling Institute of Medical Research, Sydney, New South Wales, Australia

${ }^{14}$ Mid North Coast Cancer Institute, Coffs Harbour Hospital, Coffs Harbour, New South Wales, Australia

${ }^{15}$ Illawarra Shoalhaven Cancer and Haematology Network, Wollongong Hospital, Wollongong, New South Wales, Australia 
Acknowledgements Special study personnel, patients and their families.

Contributors PG, PSH, MS, NL, JS, IM, I0, DJA, CG and ACK were responsible for study concept (grant authors). PG, PSH, MS, NL, JS, IM, IO, DJA, CG, ACK, RLM and NW were responsible for protocol development. PG, AT, AJM, NW, AW, CH, ACK, MS, $\mathrm{CG}, \mathrm{PF}, \mathrm{SC}, \mathrm{KB}$ and MA were responsible for study conduct.

Funding NSW Department of Health, drug supply by Tilray.

Competing interests None declared.

Patient consent Not required.

Ethics approval This protocol was approved at the Sydney Local Health District ethics review committee (Royal Prince Alfred Hospital zone) and ethics review committees for all participating sites.

Provenance and peer review Not commissioned; externally peer reviewed.

Open access This is an open access article distributed in accordance with the Creative Commons Attribution Non Commercial (CC BY-NC 4.0) license, which permits others to distribute, remix, adapt, build upon this work non-commercially, and license their derivative works on different terms, provided the original work is properly cited, appropriate credit is given, any changes made indicated, and the use is non-commercial. See: http://creativecommons.org/licenses/by-nc/4.0/.

\section{REFERENCES}

1. Navari RM, Aapro M. Antiemetic Prophylaxis for ChemotherapyInduced Nausea and Vomiting. N Engl J Med 2016;374:1356-67.

2. Multinational Association of Supportive Care in Cancer. MASCC/ ESMO Antiemetic guideline. 2016. http://www.mascc.org/antiemeticguidelines (accessed 21 Aug 2017).

3. Roila F, Herrstedt J, Aapro M, et al. Guideline update for MASCC and ESMO in the prevention of chemotherapy- and radiotherapy-induced nausea and vomiting: results of the Perugia consensus conference. Ann Oncol 2010;21:v232-43.

4. Gilmore JW, Peacock NW, Gu A, et al. Antiemetic guideline consistency and incidence of chemotherapy-induced nausea and vomiting in US community oncology practice: INSPIRE Study. J Oncol Pract 2014;10:68-74.

5. Molassiotis A, Saunders MP, Valle J, et al. A prospective observational study of chemotherapy-related nausea and vomiting in routine practice in a UK cancer centre. Support Care Cancer 2008;16:201-8

6. Aapro M, Molassiotis A, Dicato M, et al. The effect of guidelineconsistent antiemetic therapy on chemotherapy-induced nausea and vomiting (CINV): the Pan European Emesis Registry (PEER). Ann Oncol 2012;23:1986-92

7. Frame DG. Best practice management of CINV in oncology patients: I. Physiology and treatment of CINV. Multiple neurotransmitters and receptors and the need for combination therapeutic approaches. J Support Oncol 2010;8:5-9.

8. Penington DG. Medical cannabis: time for clear thinking. Med J Aust 2015;202:74-5.

9. Kramer JL. Medical marijuana for cancer. CA Cancer J Clin 2015;65:109-22.

10. Bhattacharyya S, Morrison PD, Fusar-Poli P, et al. Opposite effects of delta-9-tetrahydrocannabinol and cannabidiol on human brain function and psychopathology. Neuropsychopharmacology 2010;35:764-74.

11. Allsop DJ, Copeland J, Lintzeris $\mathrm{N}$, et al. Nabiximols as an agonist replacement therapy during cannabis withdrawal: a randomized clinical trial. JAMA Psychiatry 2014;71:281-91.

12. Duran $M$, Pérez $E$, Abanades $S$, et al. Preliminary efficacy and safety of an oromucosal standardized cannabis extract in chemotherapy-induced nausea and vomiting. Br J Clin Pharmacol 2010;70:656-63.

13. Herzog S, Shanahan M, Grimison P, et al. Systematic Review of the Costs and Benefits of Prescribed Cannabis-Based Medicines for the Management of Chronic Illness: Lessons from Multiple Sclerosis. Pharmacoeconomics 2018;36:67-78.

14. Cancer Institute of New South Wales. Prevention of chemotherapy induced nausea and vomiting. 2017. https://www.eviq.org.au/clinicalresources/side-effect-and-toxicity-management/gastrointestinal/7prevention-of-chemotherapy-induced-nausea-and-vo (accessed 30 Oct 2017).

15. Richardson J, lezzi A, Khan MA, et al. Validity and reliability of the Assessment of Quality of Life (AQoL)-8D multi-attribute utility instrument. Patient 2014;7:85-96.

16. Campbell MK, Torgerson DJ. Bootstrapping: estimating confidence intervals for cost-effectiveness ratios. QJM 1999;92:177-82.

17. Fenwick E, O'Brien BJ, Briggs A. Cost- effectiveness acceptability curves-facts, fallacies and frequently asked questions. Health Econ 2004;13:405-15. 